\title{
Self Regulation of the Heart: Natural Frequency and Damping of the Heart Contractions
}

\author{
Homayoun Bahramali ${ }^{1}$, Dmitriy Melkonian ${ }^{* 2}$ and Oliver O’Connell ${ }^{1}$ \\ ${ }^{I}$ Bioemotec Company, 3 Prince Edward Pde, Hunters Hill, NSW 2110, Australia \\ ${ }^{2}$ Brain Dynamics Centre, Westmead Hospital, Westmead, NSW 2145, Australia
}

\begin{abstract}
ECG frequency analysis is complicated by the fact that ECG signals are non-stationary, that is, their activity patterns change slowly or intermittently as a result of variations in a number of physiological and physical influences. Previous applications of spectral methods to the ECG analysis have identified power spectrum of the intervals between heart beats, characteristic frequencies of which belong to low frequency ranges. Here we introduce a new approach, based on the recently developed SBF algorithm of numerical Fourier transform spectroscopy, which can evaluate frequency transfer functions from electrical transients induced by the heart activity. Using this approach, we found that ECG components, induced by a heart contraction are consistent with the transient behavior of a classical underdamped harmonic oscillator. Identification of this model using the Bode diagram, allows us to characterize heart contractions by the novel parameters: natural frequency and damping ratio of heart contraction. Characteristic natural frequency of a healthy heart is in the frequency range between 13 and $20 \mathrm{~Hz}$.
\end{abstract}

Keywords: ECG analysis, QRS complex, time-frequency analysis, SBF algorithm, self regulation, transfer function, Bode diagram, heart contraction, natural frequency, damping ratio.

\section{INTRODUCTION}

The human heart is so widely observed an organ that most cyberneticians are already acquainted with certain basic facts of its anatomy and physiology. In the simplest terms, the heart is a four chambered pump consisted of two ventricles and two atria which are made up of specialized muscle tissue. The muscular walls of the ventricles are capable of organized contraction to eject blood into the circulation followed by relaxation during which the atria are refilled with blood. By altering the beat dynamics, the heart manages the blood supply according to the changing physiological demands of the organism. This system of self-regulation supports our well being and is one of the most remarkable cybernetic systems observed in nature.

Self-regulation is a property of the electrical activity in the heart which initiates mechanical contraction and consequently acts as a prime mover of cardiovascular events.

Because the heart is surrounded by conduction tissue, the cardiac potentials produce changes in the distribution of potential field on the body surface. This potential can be measured from skin electrodes, and the electrical activity of the heart can be monitored by the electrocardiogram (ECG).

As a composite of many functionally meaningful components, the ECG has a potential to provide empirical basis for understanding the heart as a cybernetic system. Since the system is a "black box", a general approach to the problem is to apply the dynamic system identification using experimental records of its transient [1]. Broadly speaking, a transient

*Address correspondence to this author at the Brain Dynamics Centre, Westmead Hospital, Westmead, NSW 2145, Australia;

E-mail:dmitri@psychiat.usyd.edu.au is a signal that can be regarded as the system's response to certain input functions. The means to convert empirical transient response to equivalent frequency transfer function are provided by numerical estimation of Fourier integral transforms.

However, traditional approaches to this problem are addressed to stationary systems. By contrast, the ECG is a nonstationary signal, and the activity patterns may be significantly different from beat-to-beat. A specific aspect of this non-stationary ECG signal is that various ECG waveforms have different functional meaning, that is they should be regarded as the transients relevant to different elements of the control system. Such transients change their behavior in relatively brief intervals which may have different length. Conventional methods of digital spectral analysis, such as the Fast Fourier Transform (FFT) and autoregressive models [2], are not applicable to non-stationary processes. The attempts to support FFT applications to the ECG time series processing by simplifying assumptions, such as the proposition of the ECG periodicity [3], show that essential aspects of ECG dynamics are missing in the analysis outputs [4].

The possibility of the implementation of major classical methods of control system analysis to the study of the heart self-regulation is supported in our study by a recently developed similar basis function (SBF) algorithm which provides means for effective short time frequency analysis $[5,6]$. Using this new computational tool for time-frequency analysis of characteristic ECG segments, we found that the QRS complex of the ECG is not an integration of separate components but rather a solitary process. That is to say that QRS complex is a single process and not three separate waves, as it is conventionally regarded. We suggest that the QRS component may be regarded as an underdamped oscillation, i.e. a transient response of a classical harmonic oscillator induced 
by impulsive forcing of its input. To our knowledge this finding for the first time makes it possible to treat the self regulation of the heart in terms of empirically based frequency transfer functions and differential equations. On these grounds we introduce the new parameters, natural frequency and damping ratio of the heart contraction, and identify their values for normal conditions of heart activity.

\section{METHODS AND MATERIALS}

\section{ECG Fragmentary Decomposition}

The ECG is a non-stationary signal and for any kind of a short-time frequency analysis, it is essential to separate the signal into sections that are of similar type. We support the ECG segmentation principle by a generally accepted physiological notion of a peak as a functionally meaningful component of a mass potential, i.e. a macrophysical potential produced by multiple cellular sources (microphysical scale). This involves an understanding of a component as being not just a peak in the waveform, but a whole deflection (positive or negative) with a specific shape.

A basis for such specification of descriptive geometric elements is contained in the previous papers which suggested fragmentary decomposition method for partitioning the electrophysiological signals into sections named half waves [7, 8]. The term "half-wave" was adopted from the field of oscillatory phenomena, in which a cycle of two deflections of opposite polarities may be often regarded as a wave. In this context a single deflection is a half-wave. The half waves of an electrophysiological signal correspond to the regions over which the signal peaks are generated.

Central to the partitioning of a signal into half-waves is the estimation of segmentation points, i.e. the boundaries between segments. Let $v(t)$ represent the ECG, which is regarded as a continuous time function. We define two types of segmentation points exemplified in Fig. (1).

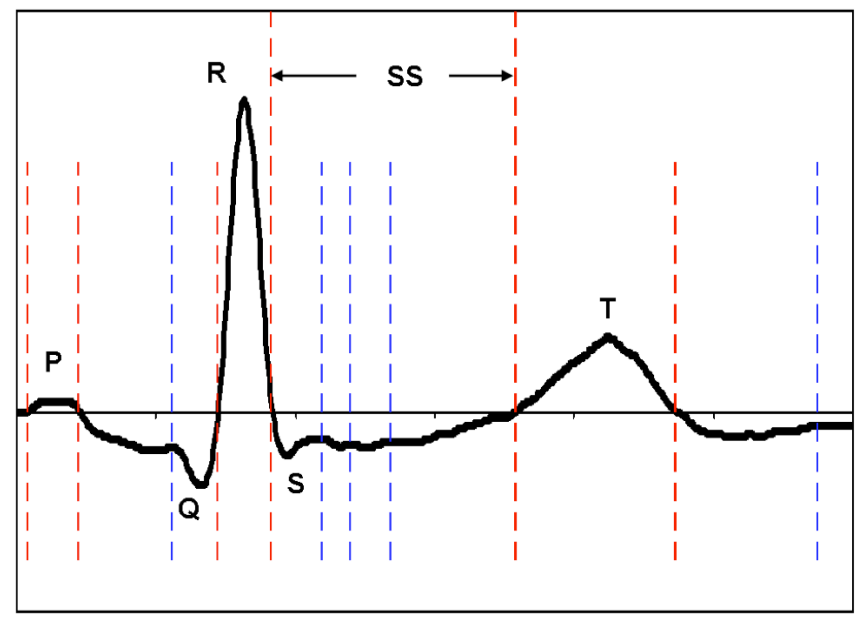

Fig. (1). Exemplifies ECG segmentation with zero-crossings shown by red vertical lines and points of minimums by blue vertical lines. P, Q, R, S and T denote conventional ECG waves. $\mathrm{SS}$ denotes the segment between zero crossings which extends from the end of R to the beginning of T.
The first is zero-crossing, i.e. a point where signal $\mathrm{v}(t)$ goes through zero crossing. The second is a point of minimum defined as a time instant where $|\mathrm{v}(t)|$ has a local minimum.

Each half-wave function (HWF) is characterized by the peak amplitude and peak latency.

\section{Fourier Transform Spectroscopy}

We use HWFs and corresponding principles of segmentation as a basis for ECG time-frequency analysis. By this we pursue two basic goals.

Firstly, we focus the procedures of time-frequency analysis on the intervals between the segmentation points. This removes uncertainty in the choice of the intervals for the short time frequency analysis and allows us to construct standard procedures of ECG digital processing. Secondly, the time domain ECG decomposition into HWFs and further time-frequency analysis of HWF ensembles provides means to analyse the ECG as a non-stationary signal using the novel model based methodology of fragmentary decomposition $[9$, $10]$.

Accordingly, the point of departure to construct algorithms of ECG short-time analysis is given by a finite exponential Fourier integral of the following form

$$
\mathrm{V}(\omega)=\int_{t_{j}}^{t_{k}} \mathrm{v}(t) \exp (-i \omega t) d t
$$

where $\mathrm{V}(\omega)$ is the complex spectrum of $\mathrm{v}(\mathrm{t})$ segment from segmentation point $\mathrm{t}_{\mathrm{j}}$ to segmentation point $\mathrm{t}_{\mathrm{k}}, i=\sqrt{-1}$ and the angular velocity $\omega$ is related to the frequency $\mathrm{f}$ by $\omega=2 \pi f$. To create universal computational schemes, we present (1) in the form

$$
\mathrm{V}(\omega)=\mathrm{U}(\omega) \exp \left(i \omega t_{j}\right) \text {, }
$$

where

$$
\begin{aligned}
& \mathrm{U}(\omega)=\int_{0}^{T} \mathrm{u}(t) \exp (-i \omega t) d t, \\
& \mathrm{u}(t)=\mathrm{v}\left(t-t_{j}\right) \text { and } T=t_{k}-t_{j} .
\end{aligned}
$$

In terms of real functions,

$\mathrm{U}(\omega)=\mathrm{U}_{C}(\omega)-i \mathrm{U}_{S}(\omega)$,

where $\mathrm{U}_{C}(\omega)$ and $\mathrm{U}_{S}(\omega)$ are the real and imaginary parts of the complex spectrum $U(\omega)$. These parts are defined by the following trigonometric integrals:

$$
\begin{aligned}
& \mathrm{U}_{\mathrm{C}}(\omega)=\int_{0}^{T} \mathrm{u}(t) \cos \omega t d t \\
& \mathrm{U}_{\mathrm{S}}(\omega)=\int_{0}^{T} \mathrm{u}(t) \sin \omega t d t
\end{aligned}
$$


Both $\mathrm{U}_{C}(\omega)$ and $\mathrm{U}_{S}(\omega)$ contain information that makes it possible to restore $u(t)$ using the following inverse Fourier cosine or sine transforms:

$$
\begin{aligned}
& \mathrm{u}(t)=\frac{2}{\pi} \int_{0}^{\infty} \mathrm{U}_{C}(\omega) \cos \omega t d \omega, \\
& \mathrm{u}(t)=\frac{2}{\pi} \int_{0}^{\infty} \mathrm{U}_{\mathrm{S}}(\omega) \sin \omega t d \omega .
\end{aligned}
$$

Given that $\mathrm{u}(\mathrm{t})$ is a signal produced by a physically realizable system, in the high frequency ranges both $\mathrm{U}_{C}(\omega)$ and $\mathrm{U}_{S}(\omega)$ have a tendency to decrease with increasing frequency. Therefore, it is always possible to find an angular frequency $\Omega$ above which the $\mathrm{U}_{C}(\omega)$ and $\mathrm{U}_{S}(\omega)$ are negligibly small. Therefore, $\mathrm{u}(\mathrm{t})$ may be found from the following approximate relationships:

$$
\begin{aligned}
& \mathrm{u}(t) \approx \frac{2}{\pi} \int_{0}^{\Omega} \mathrm{U}_{C}(\omega) \cos \omega t d \omega \\
& \mathrm{u}(t) \approx \frac{2}{\pi} \int_{0}^{\Omega} \mathrm{U}_{\mathrm{S}}(\omega) \sin \omega t d \omega
\end{aligned}
$$

Equations (2) and (3) which transform the signal segment to the frequency domain and equations (4) and (5) (the inverse problem of the frequency to time domain transformations) have the form of the following finite trigonometric integrals:

$$
\begin{aligned}
& \mathrm{Y}_{\mathrm{C}}(u)=\int_{0}^{L} \mathrm{y}(x) \cos u x d x \\
& \mathrm{Y}_{\mathrm{S}}(u)=\int_{0}^{L} \mathrm{y}(x) \sin u x d x
\end{aligned}
$$

where $\mathrm{L}$ defines the interval of integration.

Classical numerical methods that provide a maximum precision in the estimation of trigonometric integrals are based on the approximation of $\mathrm{y}(\mathrm{x})$ by algebraic polynomials [11]. Algorithmic implementations of these computational schemes using first order polynomials significantly improved accuracy of frequency domain measures, as compared with FFT, in a number of physical applications of Fourier transform spectroscopy $[12,13]$. Another important advantage is that the necessity of windows for spectral analysis is eliminated. However, basic procedures of polynomial interpolation are not supported by effective algorithms and require tedious computations. Using first order polynomials, the idea of the SBF algorithm is to decompose the piecewise-linear approximation function into the sum of finite elements (basis function) with similar spectral characteristics. This principle reduces numerical estimation of trigonometric integrals (6) and (7) to a number of relatively simple and standard time and frequency domain transformations and provides opportunity to construct efficient algorithms. See appendix for description of the SBF algorithm.

\section{The Data and Software}

ECG recordings of 40 normal subjects were studied from the PTB Diagnostic ECG Database that is available over the internet $[14,15]$. The PTB database is the source of high fidelity digital ECG records (sampling frequency of $1 \mathrm{kHz}$ ) including both normal and abnormal cardiac activity with accompanying diagnoses published in August 2004. Each recording has duration of $115.2 \mathrm{sec}$ and includes 15 simultaneously measured signals: the conventional 12 leads together with the 3 Frank lead ECGs.

We designed special software for ECG digital processing. The pre-processing stage involves a two-step algorithm of ECG artifact elimination using digital recursive Boxcar filters: 1) removing irrelevant low frequency components using high pass filter with $300 \mathrm{~ms}$ window length, 2) removing high frequency artifacts using low pass filter with $2 \mathrm{~ms}$ window length.

Major processing routines include: (1) signal adaptive segmentation using HWFs, (2) short-time spectral analysis of ECG segments between selected segmentation points, (3) the control of the accuracy of spectral characteristics using inverse Fourier transforms, (4) identification of estimated spectral profiles using as a reference standard frequency characteristics of typical elements of control systems, (5) statistical analysis of the time and frequency domain parameters of identified control systems. Numerical computations of both direct and inverse Fourie transforms are performed using the SBF algorithm.

\section{RESULTS}

\section{Distributions of HWFs}

ECG quantitative analysis is complicated by an ambiguity in the measurements of the morphology of ECG major waves which are conventionally denoted by $\mathrm{P}, \mathrm{Q}, \mathrm{R}, \mathrm{S}$ and $\mathrm{T}$ letters (Fig. 1). The understanding of the largest ECG peak between zero-crossings as a major descriptive element of the wave tells us how to measure the peak parameters of the wave. It is however, uncertain when the wave begins and ends. Zero-crossings can not serve as reliable markers of these time instants, because converging evidence from quantitative studies of ECG component composition have demonstrated the heterogeneous properties of ECG waveforms. For example, the atrial activity (AA) and ventricular late potentials (VLPs) appear to be activated essentially synchronously with the QRS complex [16, 17].

The segmentation technique of the fragmentary decomposition which uses points of minimums, in addition to zerocrossings, provides means for a more comprehensive characterization of the morphological features of ECG waveforms. To use this opportunity, we introduce the notion of a wave segment (WS), which is the segment between subsequent zero-crossings over which the wave is developed. WS is a general notation, while by PS, QS, RS, SS and TS we denote the wave segments which are relevant to conventional P, Q, R, S and T waves. Fig. (1) exemplifies the SS segment.

We use the number of HWFs identified in the WS as a measure of the complexity of the wave segment. This parameter is denoted by $\mathrm{CX}$.

The differences and profound changes in composite structure of ECG wave segments during different heart beats 
(ECG cycles) are illustrated by the histograms in Figs. (2-4). The analysis was applied to ECG records from the lead i.

Figs. $(\mathbf{2}, \mathbf{3})$ summarize the data from 40 normal subjects (PTB database).

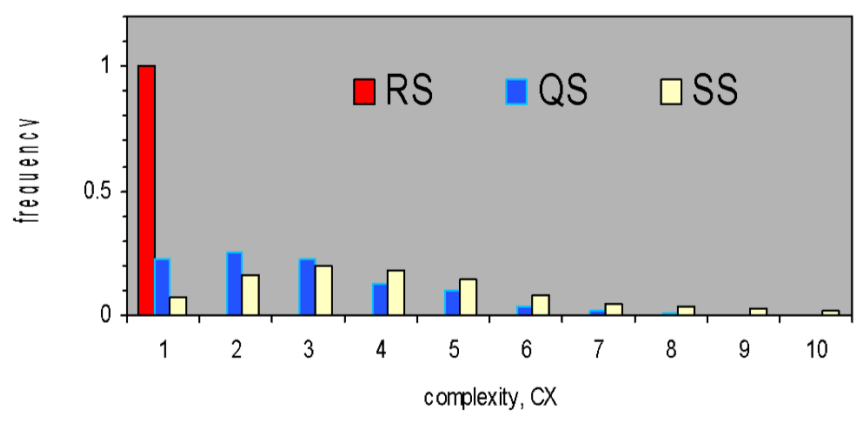

Fig. (2). Illustrates complexity of RS, QS and SS wave segments.

Essential aspect of the data displayed by the histogram in Fig. (2) is that the complexity of a wave segment typically changes from cycle to cycle. Therefore, CX is a variable. In the analyses of QS, RS and SS the range of CX was from 1 to 10 . Accordingly, the horizontal axis of the histogram is labeled with the values of complexity from 1 to 10 . For each bin the bars show the frequency with which the wave segment (QS, RS or SS) of corresponding complexity appeared in 5418 analyzed ECG cycles (ECG segments which correspond to heart beats).

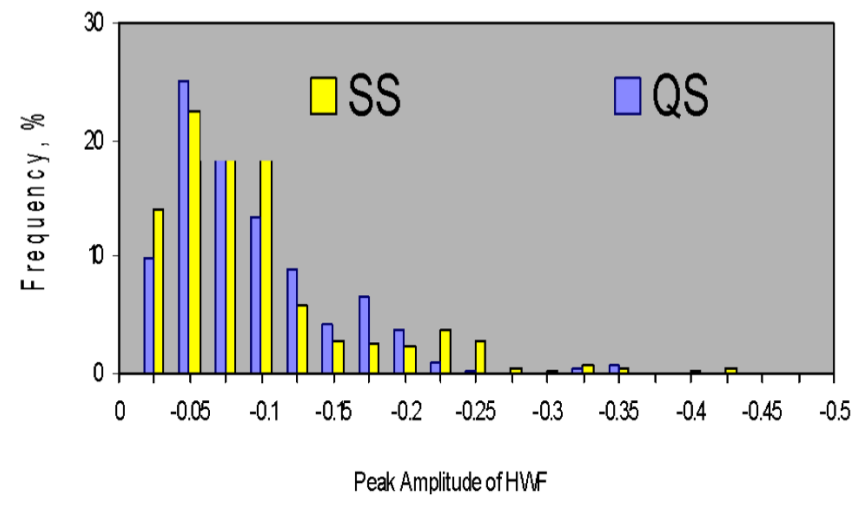

Fig. (3). Frequency distributions of the peak amplitudes of HWFs identified from SS and QS wave segments. Bin width is $0.025 \mathrm{mV}$. Total numbers of identified HWFs are: QS $-11,790$, SS - 18,375. Differences in the sample sizes are due to the differences in the complexity of QS and SS.

According to the histogram, the complexity of RS was constant with $C X=1$. This means that during all analyzed ECG cycles the R wave was developed as a monolithic component. However, it is important to note that similar analyses of ECGs from different leads and larger subjects groups (PTB database) indicate that complexity of RS is not necessarily a 1 . It may have values of 2 and even 3 . A general conclusion is that a monolithic $\mathrm{R}$ wave is a highly expected feature of ECG recorded from i lead. Thus, a unit complexity appears as an important distinguishing property of the $\mathrm{R}$ wave in normal subjects.

For QS and SS the complexity is significantly higher. A mean value of QS complexity observed in the group of 40 subjects varied from 1.05 to 3.72 . The corresponding estimates for SS were 1.01 and 6.47 , respectively.
Additional characterization of the composite nature of QS and SS is provided by the frequency distributions of the peak amplitudes of HWFs which were identified from these segments. The histograms in Fig. (3) indicate a large proportion of low amplitude HWFs the absolute value of the peak amplitude of which is below $0.2 \mathrm{mV}$. Only high fidelity ECG records provide means to detect these components.

Some insights into the nature of the variability of HWF amplitudes came from the analysis of the data from individual subjects. A striking feature of the frequency distributions in Fig. (4) is a clear indication of two distinct groups of components for both QS and RS.

Given QS, the frequency distribution is divided into the two groups by the vertical red line. The first group includes HWFs with relatively large amplitudes the modules of which are in the range from 0.13 to $0.175 \mathrm{mV}$. The amplitude range for the second group is between -0.01 to $-0.125 \mathrm{mV}$. The presence of two distinct distributions emphasized by the vertical green lines is even more pronounced for SS.

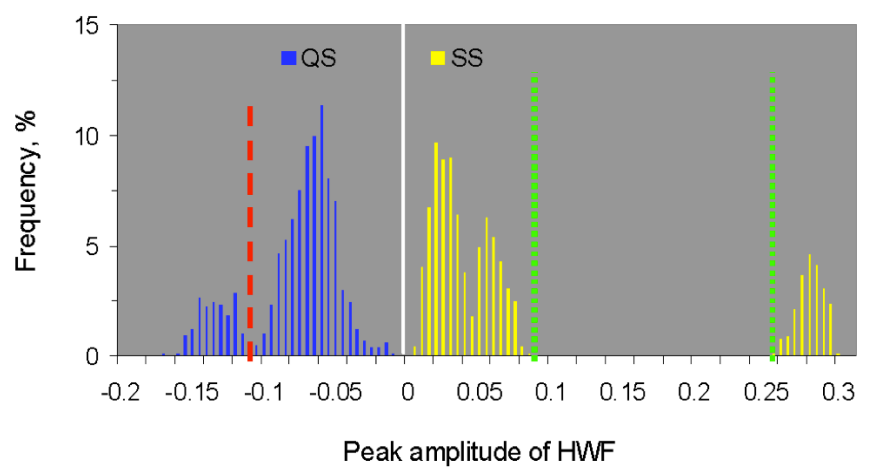

Fig. (4). Illustrates frequency distributions of the peak amplitudes of HWFs identified from ECG of a single subject. The histogram for SS deals with the modules of peak amplitudes. Bin width is $0.005 \mathrm{mV}$.

Such clear separation of low and high amplitude HWFs was seen in approximately $50 \%$ of ECGs. The major factor which makes the differences less pronounced in the remaining cases is the temporal overlap of ECG components.

A composite nature of ECG signal on the Q and S segments may typically be recognized by double peak waveforms of average ECGs in QS and SS segments (Fig. 5).

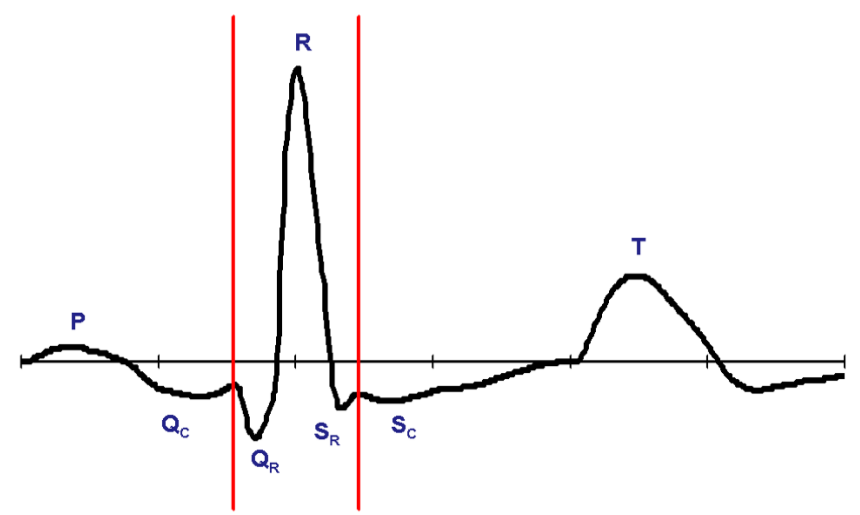

Fig. (5). Illustrates composite nature of the waveform of average ECG on the wave segments QS and SS. Vertical red lines show the boundaries between $\mathrm{Q}_{C}-\mathrm{Q}_{\mathrm{R}}$ and $\mathrm{S}_{\mathrm{R}}-\mathrm{S}_{\mathrm{C}}$, respectively. 
To differentiate these components from conventional $\mathrm{Q}$ and $\mathrm{S}$, we introduce the notations $\mathrm{Q}_{\mathrm{C}}, \mathrm{Q}_{\mathrm{R}}, \mathrm{S}_{\mathrm{R}}$ and $\mathrm{S}_{\mathrm{C}}$, which are explained by Fig. (5). Conceptually, $Q_{R}$ and $S_{R}$ may be regarded as synonymous to conventional $\mathrm{Q}$ and $\mathrm{R}$. However, having in mind the major findings of this study, we use subscript " $R$ " in $Q_{R}$ and $S_{R}$ in order to emphasize that these components have common origins with the $\mathrm{R}$. In the histogram of Fig. (4) these components correspond to the groups of HWFs with larger absolute amplitudes. The frequencies of $\mathrm{Q}_{\mathrm{R}}$ and $\mathrm{S}_{\mathrm{R}}$ indicate that these entities are monolithic components. By contrast, $\mathrm{Q}_{\mathrm{C}}$ and $\mathrm{S}_{\mathrm{C}}$ are composite components (subscripts "C" stand for Composite).

\section{FREQUENCY DOMAIN CHARACTERISTICS OF $\mathbf{Q}_{\mathrm{R}} \mathbf{R S}_{\mathrm{R}}$}

Numerical Fourier transform spectroscopy using SBF algorithm was applied to different EEG segments including separate PS, QS, RS, SS and TS, the entire QRS complex (QS+RS+SS), etc.

The accuracy of computations was tested by the restoration of original time series from imaginary or real frequency characteristic using the same SBF algorithm. The results of a typical test are illustrated by Figs. $(6,7)$.

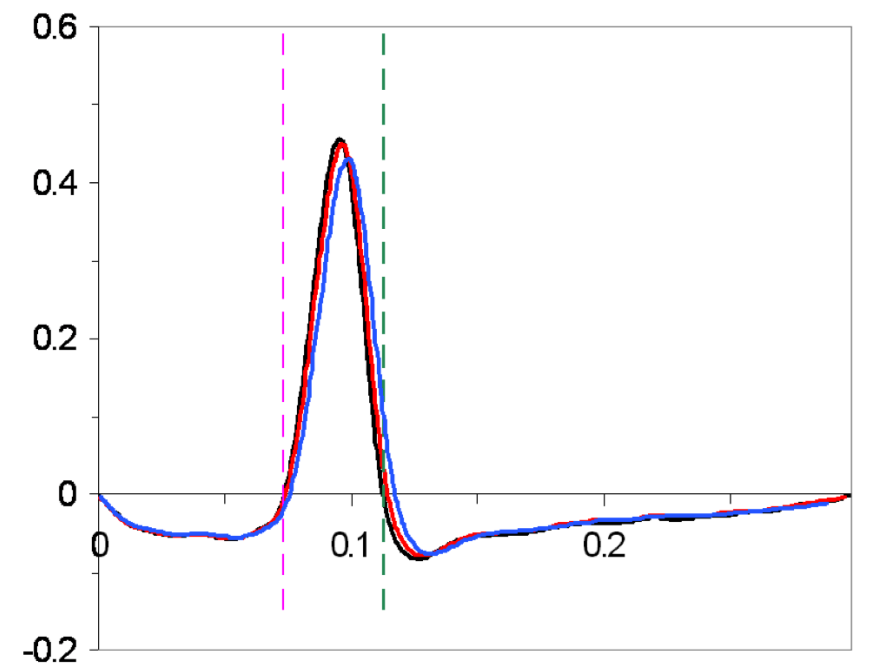

Fig. (6). The black line shows ECG waveform on the wave segment which includes QS, RS and SS. The red and blue lines show ECG waveforms which were restored from the frequency domain characteristics using the inverse Fourier transform.

The ECG segment in Fig. (6) consists of 299 samples (sampling interval $1 \mathrm{~ms}$ ). The amplitude, real and imaginary frequency characteristics computed from this 299 point time series are displayed in Fig. (7).

The accuracy of computations was tested using the inverse sine Fourier transform (equation 5). We applied inverse transform to the two sets of frequency domain samples of $U_{S}(2 \pi f)$ in the interval of frequency, f, from 1 to $100 \mathrm{~Hz}$ (logarithmic scale). First, 101 sample, i.e. 50 samples per decade. The corresponding restored time domain function is shown by the blue line. Secondly, 201 sample, i.e. 100 samples per decade. Restored function is shown by the red line. It is practically indistinguishable from the original waveform. This level of accuracy of the time to frequency domain and vice versa computations is characteristic for computational procedures which were used for the identification of frequency transfer functions.

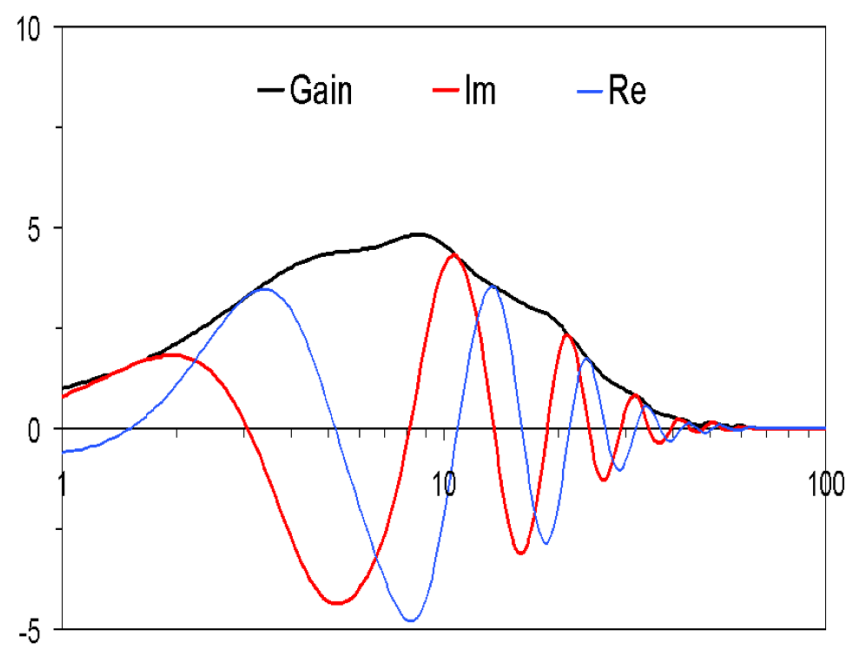

Fig. (7). Illustrates amplitude frequency characteristic (Gain), imaginary (Im) and real (Re) frequency characteristics computed from ECG waveform shown in Fig. (6) by the black line. The $\mathrm{x}$ axis unit is frequency, Hz. The y-axis units are mVs..

In the context of the control mechanisms analysis, we focus in our report on the analysis of ECG segments from the beginning of the $Q_{R}$ wave to the end of $S_{R}$ wave, i.e. the $\mathrm{Q}_{\mathrm{R}} \mathrm{RS}_{\mathrm{R}}$ complex. A major challenge in such an approach is a difficulty in an unambiguous estimation of the corresponding ECG segment. The possibility of a reliable choice has been supported by average ECGs such as shown in Fig. (5). The boundaries of the segment (vertical red lines in Fig. (5)) define the window for a short time spectral analysis. The length of the window depends on the morphology of the ECG cycle under the analysis. Thus, we essentially deal with the windows of different length.

The basic aim of the short time frequency analysis of such ECG segments was to capture and identify the dynamic properties of the waveform regarded as a transient response of a dynamic system. In this context, the frequency domain characteristics have many useful properties including a well developed general theory [18]. Our approach was to adapt the theory of frequency domain methods to define the $\mathrm{Q}_{\mathrm{R}} \mathrm{RS}_{\mathrm{R}}$ complex in terms of an adequate transfer function and differential equation.

Blue lines in Figs. $(\mathbf{8 , 9})$ show amplitude frequency characteristic (AFC) and Bode diagram computed from $\mathrm{Q}_{\mathrm{R}} \mathrm{RS}_{\mathrm{R}}$ complex of ECG. A major descriptive feature of the AFC characteristic is a resonant peak.

An important feature of the Bode diagram is that in the range of high frequencies $(>20 \mathrm{~Hz})$ the plot can be approximated by a straight line with a slope of $-40 \mathrm{~dB} /$ decade. That is, for every factor of 10 increase in frequency, the magnitude drops by $40 \mathrm{~dB}$. This feature is characteristic for a system described by a second order differential equation.

The profiles of the gain characteristic and Bode diagram are typical for simple harmonic oscillator the equation of which has the following form [19]: 
$\frac{\mathrm{d}^{2} \mathrm{y}}{\mathrm{dt}^{2}}+2 \zeta \omega_{0} \frac{\mathrm{dy}}{\mathrm{dt}}+\omega_{0}^{2} \mathrm{y}=0$

where $\omega_{0}=2 \pi f_{0}$ is the natural angular frequency and $\zeta$ is the damping ratio (factor) of the system.

The general form of the system's transfer function using the complex variable $s=i \omega$ is

$\mathrm{H}(\mathrm{s})=\frac{\omega_{0}^{2}}{\mathrm{~s}^{2}+2 \zeta \omega_{0} \mathrm{~s}+\omega_{0}^{2}}$,

with $0<\zeta<1$ and $\omega_{0}>0$.

The AFC is defined as the module of transfer function in the form

$$
|H(j \omega)|=\frac{1}{\sqrt{\left(1-\left(\frac{\omega}{\omega_{0}}\right)^{2}\right)^{2}+\left(\frac{2 \zeta \omega}{\omega_{0}}\right)^{2}}} .
$$

Accordingly the Bode diagram is expressed in decibels as

$|B(j \omega)|=-20 \log _{10}\left(\sqrt{\left(1-\left(\frac{\omega}{\omega_{0}}\right)^{2}\right)^{2}+\left(\frac{2 \zeta \omega}{\omega_{0}}\right)^{2}}\right)$

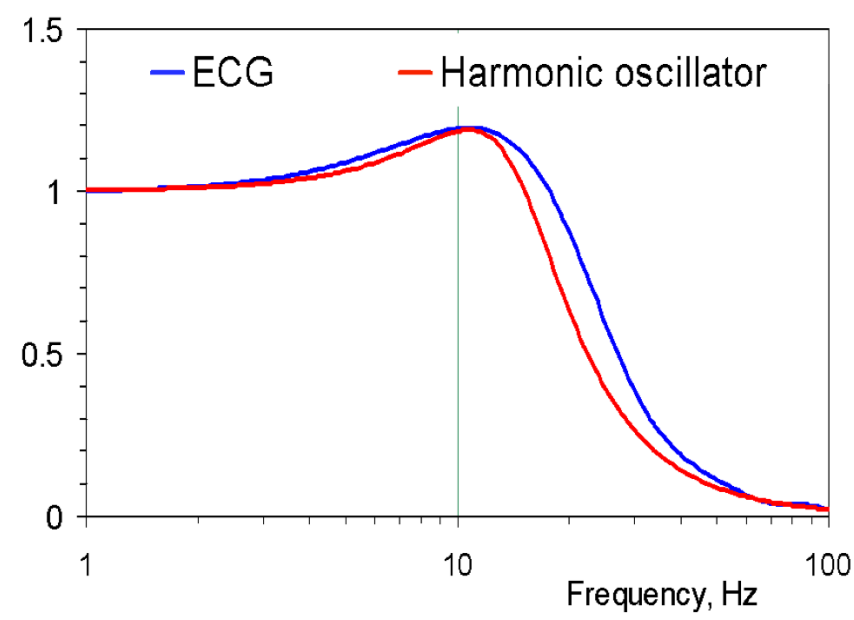

Fig. (8). Empirical (ECG) and analytical (harmonic oscillator) AFCs. The y axis is in dimensionless units.

To compare these analytical frequency dependencies with empirical AFC and Bode diagram we need empirical estimates of $\zeta$ and $\omega_{0}$ parameters. The presence of characteristic resonant peak in the $\mathrm{AFC}$ indicates that parameters should be relevant to an underdamped system.

Taken this assumption, the parameters are estimated from the following relationships:

$$
\begin{aligned}
& \omega_{\mathrm{r}}=\omega_{0} \sqrt{1-2 \zeta^{2}}, \\
& \mathrm{M}_{\mathrm{r}}=\frac{1}{2 \zeta \sqrt{1-\zeta^{2}}},
\end{aligned}
$$

where $\omega_{\mathrm{r}}$ and $\mathrm{M}_{\mathrm{r}}$ are the parameters of the resonant peak of the gain function. $\omega_{\mathrm{r}}=2 \pi \mathrm{f}_{\mathrm{r}}$ is the resonant angular frequency and $M_{r}$ is the magnitude of the resonant peak. For the AFC in
Fig. (8) $\mathrm{M}_{\mathrm{r}}=1.19$ and $\mathrm{f}_{\mathrm{r}}=10.7 \mathrm{~Hz}$. The corresponding parameters of the system are: $\mathrm{f}_{0}=14.6 \mathrm{~Hz}$ and $\zeta=0.48$. Analytical gain characteristic and Bode diagram with these parameters are shown in Figs. $(\mathbf{8 , 9})$ by the red lines. It is important to note that reasonably accurate fits of theoretical dependencies to empirical curves for a relatively large frequency diapason from 1 to 100 is supported by equality of just two parameters, $\mathrm{f}_{0}$ and $\zeta$. Such agreement is only possible if both systems are described by one and the same equation. This shows that waveform of $\mathrm{Q}_{\mathrm{R}} \mathrm{RS}_{\mathrm{R}}$ complex may be regarded as a transient response of underdamped harmonic oscillator to the application of impulse function to its input.

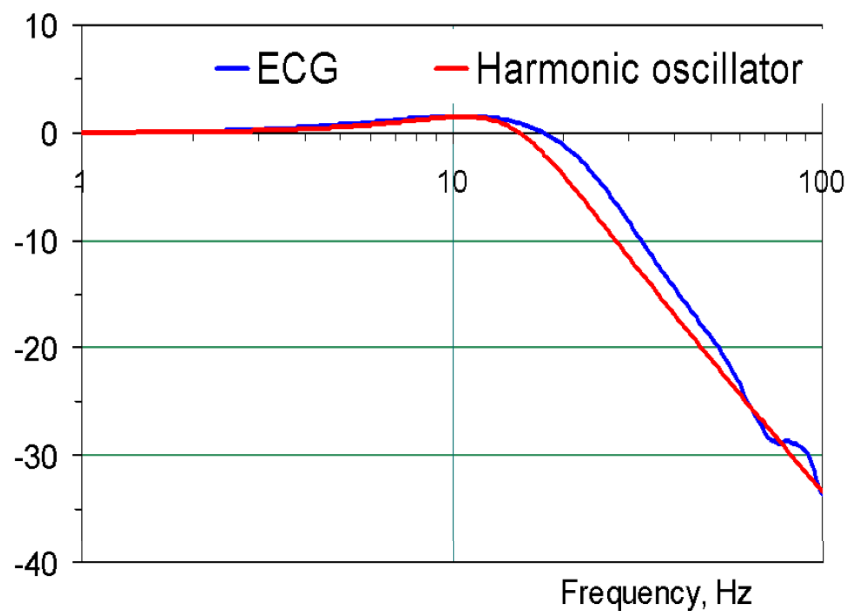

Fig. (9). Empirical (ECG) and analytical (harmonic oscillator) Bode diagrams. The y-axis units are decibels.

Similar procedures of numerical Fourier transform spectroscopy and parameter identification have been applied to $\mathrm{Q}_{\mathrm{R}} \mathrm{RS}_{\mathrm{R}}$ complexes corresponding to different heart beats. The beginning of $Q_{R}$ and the end of $S_{R}$ were taken as the first segmentation point before RS and the first segmentation point after RS, respectively.

Fig. (10) shows the gain characteristics computed from successive $Q_{R} R_{R}$ complexes for 20 successive heart beats. The analysis of relative contributions of different segments into the spectrum, revealed that a fair reproducibility of the shapes of AFCs is due to a relatively high stability of the shapes of the $\mathrm{R}$ wave during different beats. However, the parameters of AFC peaks show significant deviations from mean values. The comparison of AFC profiles with their time domain counterparts indicates that the variability is mainly due to the differences in ECG shapes before and after the $\mathrm{R}$ wave. The histograms in Figs. $(\mathbf{2}, \mathbf{3})$ show that complexity of ECG waveforms in these regions undergoes remarkable changes from beat to beat. These alterations change the profiles and temporal overlap of ECG components which develop on QS and SS.

The possibility of reliable estimation of defined $\mathrm{Q}_{\mathrm{R}}$ and $S_{R}$ waveforms depends on the character of ECG waveform morphology during different beats. In some cases, the identification of $Q_{R}$ and $S_{R}$ is relatively simple. However, the temporal overlap of ECG components often obscures these waves. Such situation is the reflection of the ECG nonstationarity, and consequently the heterogeneous character of 
the sources of ECG waveforms. Universal solutions of the problem necessitate new approaches.

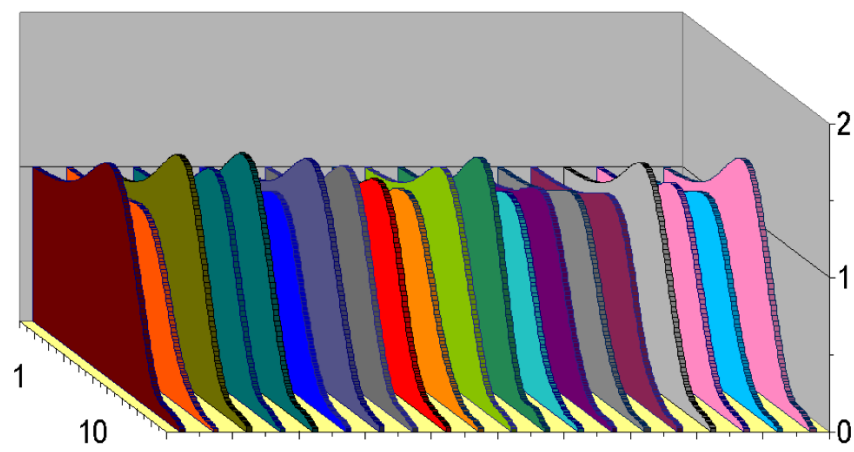

Fig. (10). AFCs for 20 successive beats. The abscissa scale shows frequencies from 1 to $100 \mathrm{~Hz}$. The vertical axis is in dimensionless units.

We found a reasonably accurate solution using the average ECG as an empirical template for the estimation of the boundaries of the $\mathrm{Q}_{\mathrm{R}} \mathrm{RS}_{\mathrm{R}}$ complex. We are planning to describe this technique in the following publications. Here we note that though the template based identification of the $\mathrm{Q}_{\mathrm{R}} \mathrm{RS}_{\mathrm{R}}$ complexes reduces the beat-to-beat parameter variability, it is clear that important aspect of the heart selfregulation are governed by probabilistic laws.

\section{OPTIMUM CONDITIONS OF HEART CONTRAC- TIONS}

The analysis of the ECG data from the whole subject group revealed that the consistency with which the harmonic oscillator reproduces electrical transients induced by heart contractions is different for different ranges of the damping ratio. Fig. (11) exemplifies the character of a typical deviation of analytical AFC from empirical AFC in the case of a low damping ratio.

We found that the harmonic oscillator predicts with fair accuracy the time and frequency domain characteristics of the $\mathrm{Q}_{\mathrm{R}} \mathrm{RS}_{\mathrm{R}}$ complex if the damping ratio is about 0.38 or higher. Decrease of the damping ratio below this level increases divergence of the model from empirical dependences.

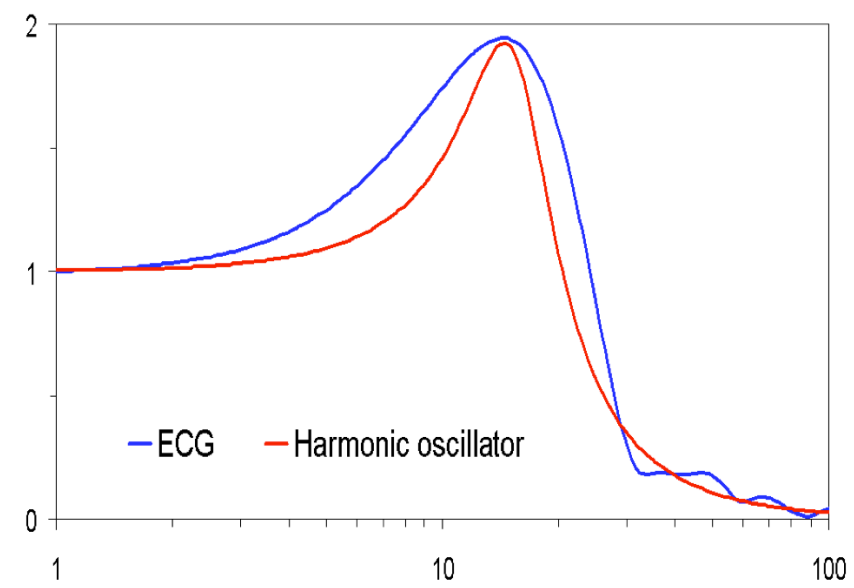

Fig. (11). Gain function of $\mathrm{Q}_{\mathrm{R}} \mathrm{RS}_{\mathrm{R}}$ complex with identified damping ratio 0.270 . Peak amplitude $=1.94$, peak frequency $=14.5 \mathrm{~Hz}$, natural frequency $=15.6 \mathrm{~Hz}$.
The deviation may be caused by different factors. The analysis of a general character of the changes in the frequency domain profiles of AFCs and Bode functions allows us to link the deviation with the character of the forcing function. The proposition is illustrated by the block scheme shown in Fig. (12).

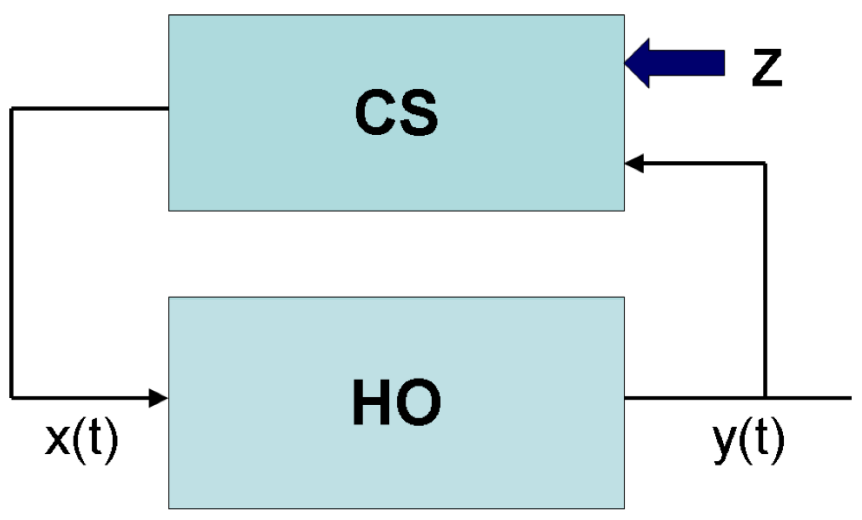

Fig. (12). Elements of the system of self regulation.

The HO is a harmonic oscillator the input and output functions of which are $x(t)$ and $y(t)$, respectively. The complex spectra of these functions, $X(\omega)$ and $Y(\omega)$, are connected in the frequency domain by the relationship,

$\mathrm{Y}(\omega)=\mathrm{H}(\omega) \mathrm{X}(\omega)$.

The input function is produced by the control system CS which receives information via input functions $\mathrm{y}(\mathrm{t})$ and $\mathrm{Z}$. $y(t)$ transmits results of the heart contraction. $Z$ transmits messages from a number of sources involved into the control of heart activity.

We presume that under normal resting conditions the $\mathrm{HO}$ is an underdamped harmonic oscillator and $\mathrm{x}(\mathrm{t})$ is a short impulse regarded as a Dirac delta function. The complex spectrum of $x(t)$ is $X(\omega)=1$. Consequently,

$\mathrm{Y}(\omega)=\mathrm{H}(\omega)$.

This means that frequency spectrum of $\mathrm{Q}_{\mathrm{R}} \mathrm{RS}_{\mathrm{R}}$ complex is consistent with the frequency spectrum of a harmonic oscillator. We may regard frequency functions in Figs. $(\mathbf{8 , 9})$ as an example of this condition. Accordingly, $\zeta=0.48$ and $\mathrm{f}_{0}=14.6 \mathrm{~Hz}$ may be regarded as typical parameters for normal conditions.

We associate abnormal condition with the increase of the peak amplitude of the gain function. However, such an increase does not affect the slope of Bode diagram in the high frequency range. It remains at the level of $40 \mathrm{~dB}$ drop per decade. Such dependency is characteristic for a second order differential equation, such as the equation of a harmonic oscillator (8). This fact allows us to associate abnormal conditions with distortions of the form of input function $\mathrm{x}(\mathrm{t})$.

Physical support for such proposition may be quite simple. It is well established that self regulation of the heart is supported by coordinated activity of multiple cardiac fibers and nerve cells. In this context, a precise initiation of the heart contraction may demand a highly synchronous coordination of information processing and control elements. 
Different forms of the loss of synchronicity may distort the quality of forcing. In terms of our model, we regard this process as prolongation and disfigure of input function. Therefore, the frequency domain characteristics of the $\mathrm{Q}_{\mathrm{R}} \mathrm{RS}_{\mathrm{R}}$ complex are now dependent on both the parameters of the harmonic oscillator and the form of the forcing function. Such interplay of different factors is likely to underlie the departure of empirical frequency dependencies from a simple model of harmonic oscillator. Therefore, the simulation of abnormal conditions demands the complication of the model and, accordingly, the introduction of additional parameters. We don't tackle this modeling problem in the present paper. However, we use these ideas to suggest a novel concept of optimal conditions of heart contractions. We define optimal conditions of the heart contraction as those under which the dynamics of contraction shows the best agreement with underdamped harmonic oscillation, i.e. is described by a minimum number of parameters. Following to this idea, we found that optimal conditions of the heart contractions are reflected by the damping ratio, $\zeta$, the value of which is about $0.45-0.48$ or higher.

\section{COMMENT}

Historically, the ECG has been conceptualized as a relatively stable conglomerate of the major $\mathrm{P}, \mathrm{Q}, \mathrm{R}, \mathrm{S}$ and $\mathrm{T}$ waves which reflect the various phases of temporal and spatial distributions of ionic currents that cause the cardiac fibers to contract and subsequently relax. This outlook has shaped virtually every aspect of ECG quantitative analysis, from the types of explanations proposed for the functional significance of the waves, to the measurement nomenclature which includes the following functional entities.

- P-wave: A small low-voltage deflection away from the baseline caused by the depolarization of the atria associated with atrial contraction.

- QRS-complex: The largest-amplitude portion of the ECG produced by the activation of the ventricular mass during contraction.

- T-wave: Ventricular repolarization, whereby the cardiac muscle is prepared for the next cycle of the ECG.

- PQ-interval: The time between the beginning of atrial depolarization and the beginning of ventricular depolarization.

- $\quad$ RR-interval: The time between successive R-peaks.

- $\quad$ QT-interval: The time between the onset of ventricular depolarization and the end of ventricular repolarization.

- $\quad$ ST-interval: The time between the end of S-wave and the beginning of T-wave.

The choice of these quantities is supported by heuristic rather than theoretical criteria. Though the parameters are convenient for simple measurements based on visual ECG analysis, an obvious drawback is that they do not provide measures of the morphology of ECG waveforms. Particularly, an exact definition of the intervals is quite uncertain.
Our major finding is that a classical underdamped harmonic oscillator is a gold standard and remarkably accurate model for conceptual and quantitative analysis of the ECG components induced by the heart contraction. To our knowledge, these essential aspects of ECG dynamics are for the first time described by an empirically grounded mathematical model. This novel model predicts with fair accuracy many of the electrical properties of the heart activity using the two major parameters: natural frequency of heart contraction, $\omega_{0}$, and damping ratio of heart contraction, $\zeta$.

Consideration of the frequency transfer functions for identified sets of these parameters allowed us to define optimal conditions of the heart contraction as those under which the dynamics of contraction shows the best agreement with underdamped harmonic oscillation, i.e. is described by a minimum number of parameters.

It is tempting to speculate that the analysis of the regimes of heart contractions which are outside of the optimal conditions may provide important insights into the extreme and pathological conditions of the heart self regulation.

\section{APPENDIX}

The SBF algorithm is a method for numerical estimation of finite trigonometric integrals:

$$
\begin{aligned}
& \mathrm{Y}_{\mathrm{C}}(u)=\int_{0}^{L} \mathrm{y}(x) \cos u x d x, \\
& \mathrm{Y}_{\mathrm{S}}(u)=\int_{0}^{L} \mathrm{y}(x) \sin u x d x,
\end{aligned}
$$

where $\mathrm{Y}_{\mathrm{C}}(\mathrm{u})$ and $\mathrm{Y}_{\mathrm{S}}(\mathrm{u})$ are finite cosine and sine Fourier transforms of $y(x)$ and $[0, L]$ is the interval of integration.

The integrals of these types have a wide spectrum of applications in analysis of physical and biological dynamic systems.

Classical algorithms that provide a maximum precision in the estimation of trigonometric integrals are based on the approximation of $\mathrm{y}(\mathrm{x})$ by algebraic polynomials. Conventional procedures consist of interval by interval integration which needs tedious computations. Using first order polynomials, the idea of the SBF algorithm is to decompose the piecewise-linear approximation function into the sum of finite elements (basis function) with similar form of spectral characteristics. This principle reduces numerical integration to a number of relatively simple standard the time and frequency domain transformations and provides opportunity to construct efficient algorithms.

We presume that $\mathrm{y}(\mathrm{x})$ is represented in the interval $[0, \mathrm{~L}]$ by its discrete samples $\mathrm{y}_{\mathrm{i}}=\mathrm{y}\left(\mathrm{x}_{\mathrm{i}}\right)$ where $\mathrm{i}$ takes values from 0 to $\mathrm{N}-1$. The first sample is at $\mathrm{x}_{0}=0$, while the following samples may be chosen arbitrary following the condition $\mathrm{x}_{\mathrm{m}}>\mathrm{x}_{\mathrm{n}}$ if $\mathrm{m}>\mathrm{n}$. The last sample $\mathrm{x}_{\mathrm{N}-1}=\mathrm{L}$.

The approximating function, $\mathrm{h}(\mathrm{x})$, is created as a linear polynomial passing through $\mathrm{N}$ nodal points according to the interpolation condition:

$h_{i}=y_{i} \quad$ for $\quad i=0, . ., N-1$, 
where $\mathrm{h}_{\mathrm{i}}=\mathrm{h}\left(\mathrm{x}_{\mathrm{i}}\right)$ and $\mathrm{y}_{\mathrm{i}}$ is a nodal point.

In the interval $\left[\mathrm{x}_{\mathrm{i}}, \mathrm{x}_{\mathrm{i}+1}\right]$,

$\mathrm{h}(t)=y_{i}+\left(\Delta y_{i+1} / \Delta x_{i+1}\right) \cdot x$

where $\Delta \mathrm{y}_{\mathrm{i}+1}=\mathrm{y}_{\mathrm{i}+1}-\mathrm{y}_{\mathrm{i}}$ and $\Delta \mathrm{x}_{\mathrm{i}+1}=\mathrm{x}_{\mathrm{i}+1}-\mathrm{x}_{\mathrm{i}}$.

Fig. (13) shows the principle of piecewise-linear approximation. Given $\mathrm{y}(\mathrm{x})$ (blue line), the approximating function, $\mathrm{h}(\mathrm{x})$, is the broken line created by joining the nodal points $0,1,2,3$ and 4 by the straight lines. In the same fashion the approximation can be continued for any number of the following nodal points.

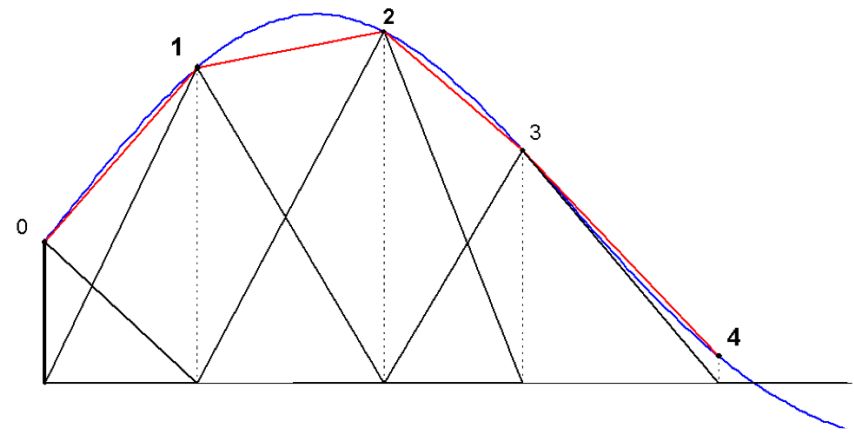

Fig. (13). Illustrates how the piecewise-linear approximating function (red line) may be represented as a sum of triangle elements.

The essence of the SBF algorithm is that $\mathrm{h}(\mathrm{x})$ is further represented by the weighted sum of self-similar functions of the following form

$\mathrm{h}(x)=\sum_{j=0}^{N-1} a_{j} \mathrm{r}\left(x / x_{j+1}\right)$,

where $a_{j}$ is a weighting coefficient and $r(x)$ is a rectangle basis element $(\mathrm{RBE})$. It is defined as $\mathrm{r}(\mathrm{x})=1-\mathrm{x}$ in the interval $[0,1]$ and $r(x)=0$ outside of this interval.

The set of self-similar functions is created in such a way that each term in the sum, $a_{j} \mathrm{r}\left(x / x_{j+1}\right)$, represents rescaled RBE. Therefore, RBE may be regarded as a building block for the construction of approximating function. In this context an important condition is that

$\mathrm{r}\left(x_{m} / x_{n}\right)=0$ for $m \geq n$.

This property of RBE supports the following simple procedure for the estimation of weighting coefficients.

Insertion of $\mathrm{x}=\mathrm{x}_{\mathrm{i}}$ and $\mathrm{h}\left(\mathrm{x}_{\mathrm{i}}\right)=\mathrm{y}_{\mathrm{i}}$ into (A3) gives the following form to the interpolation condition

$y_{i}=\sum_{j=i}^{N-1} a_{j} \mathrm{r}\left(x_{i} / x_{j+1}\right)$.

The set of such conditions for each nodal point composes a system of $\mathrm{N}$ linear equations. To deal with the system using conventional matrix notation, we collect the signal samples and weighting coefficients into N-by-1 vectors $\mathbf{y}=\left[y_{0}, . ., y_{N-1}\right]$ and $\mathbf{a}=\left[a_{0}, . ., a_{N-1}\right]$. In these terms the whole system of linear equations is $\mathbf{y}=\mathbf{U} \cdot \mathbf{a}$, where $\mathbf{U}$ is a square $\mathrm{N}-$ by- $\mathrm{N}$ matrix the entry of which in the $i$ th row and $j$ th column is $u_{i j}=\mathrm{r}\left(x_{i} / x_{j+1}\right)$. According to (A4), $u_{i j}=0$ for $i>j$, i.e. the entries of $\mathbf{U}$ below the main diagonal are zero. Therefore, $\mathbf{U}$ is upper triangle matrix. The inverse $\mathbf{V}=\mathbf{U}^{-1}$ is also an upper triangle matrix which provides the following matrix equation for the estimation of weighting coefficients from signal samples:

$\mathbf{a}=\mathbf{V} \cdot \mathbf{y}$.

To specify elements of $\mathbf{V}$, we denote the entry of the matrix in the row ' $i$ ' and the column ' $j$ ' as $v_{i j}$. The number of non-zero elements in the rows from 0 to N-3 is 3 . The formula for weighting coefficients $\mathrm{a}_{\mathrm{i}}$ for these rows is

$a_{i}=v_{i i} y_{i}-v_{i(i+1)} y_{i+1}+v_{i(i+2)} y_{i+2}$,

where

$\mathrm{v}_{\mathrm{ii}}=\mathrm{x}_{\mathrm{i}+1} / \Delta \mathrm{x}_{\mathrm{i}+1}, \mathrm{v}_{\mathrm{i}(\mathrm{i}+1)}=\mathrm{x}_{\mathrm{i}} \frac{\Delta \mathrm{x}_{\mathrm{i}+1}+\Delta \mathrm{x}_{\mathrm{i}}}{\Delta \mathrm{x}_{\mathrm{i}+1} \Delta \mathrm{x}_{\mathrm{i}}}$,

$\mathrm{v}_{\mathrm{i}(\mathrm{i}+2)}=\mathrm{x}_{\mathrm{i}-1} / \Delta \mathrm{x}_{\mathrm{i}}, \quad \Delta \mathrm{x}_{\mathrm{i}}=\mathrm{x}_{\mathrm{i}}-\mathrm{x}_{\mathrm{i}-1}$.

The last two coefficients are defined as

$\mathrm{a}_{\mathrm{N}-2}=\mathrm{v}_{(\mathrm{N}-2)(\mathrm{N}-2)} \mathrm{y}_{\mathrm{N}-2}-\mathrm{v}_{(\mathrm{N}-2)(\mathrm{N}-1)} \mathrm{y}_{\mathrm{N}-1}$,

$\mathrm{a}_{\mathrm{N}-1}=\mathrm{v}_{(\mathrm{N}-1)(\mathrm{N}-1)} \mathrm{y}_{\mathrm{N}-1}$.

The geometric principle found useful in this connection may be seen by reference to Figs. $(\mathbf{1 3}, \mathbf{1 4})$. Fig. (13) links each of nodal points from 0 to 3 with particular triangle. The nodal point 0 is the top of the rectangle which corresponds to the first term in (A3), i.e. $a_{0} r\left(x / x_{1}\right)$. A form of remaining triangles is similar to the triangle abc, shown in Fig. (14). The triangle is the sum of RBEs ohc, ofd and oga. Therefore, three RBEs are sufficient to define a particular sample of the function under the approximation.

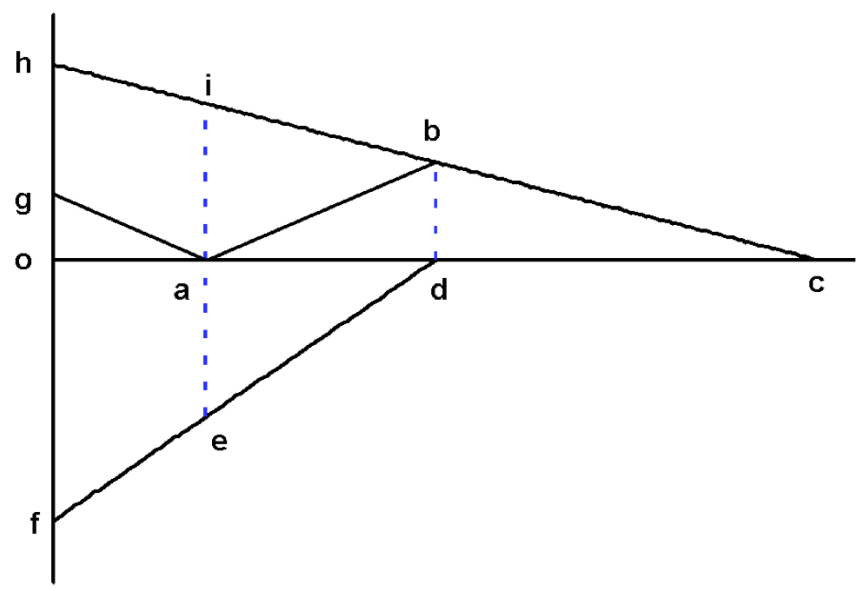

Fig. (14). Shows that triangle abc is the sum of RBEs ohc, ofd and oga.

Now that the approximating function (A3) is defined by weighting coefficients, we turn to the matter of finding the Fourier transform of $\mathrm{h}(\mathrm{x})$. Fourier cosine- and sine- integrals 
of RBE obtained by insertion of $\mathrm{r}(\mathrm{x})$ into (A1) and (A2) with $\mathrm{L} \geq 1$ are as follows:

$\mathrm{R}_{C}(u)=\frac{1-\cos u}{u^{2}}$

and

$R_{S}(u)=\frac{u-\sin u}{u^{2}} . \quad(A 6)$

Given self-similarity of basis functions in (A3), the multiplication and scaling theorems of the theory of Fourier analysis reduce the cosine- and sine- Fourier integrals from $h(t)$ to the following transforms of functions (A5) and (A6):

$$
\begin{aligned}
& \mathrm{H}_{\mathrm{C}}(u)=\sum_{n=0}^{N-1} a_{n} x_{n+1} \mathrm{R}_{\mathrm{C}}\left(u x_{n+1}\right), \\
& \mathrm{H}_{\mathrm{S}}(u)=\sum_{n=0}^{N-1} a_{n} x_{n+1} \mathrm{R}_{\mathrm{S}}\left(u x_{n+1}\right) .
\end{aligned}
$$

These equations deal with weighted sums of samples of relatively simple non-periodic functions $\mathrm{R}_{\mathrm{C}}(u)$ and $\mathrm{R}_{\mathrm{S}}(u)$.

In summary, digital Fourier transform spectroscopy using the SBF algorithm consists of two stages: estimation of interpolation coefficients from discrete samples of the signal, and evaluation of Fourier transforms at defined frequencies.

The SBF algorithm is a universal tool of numerical Fourier transform spectroscopy. The broadest conclusions to be drawn from comparison of the SBF algorithm with conventional discrete Fourier transform are as follows.

- $\quad$ The SBF algorithm provides continuous Fourier spectrum instead of discrete spectrum defined by the discrete Fourier transform.

- The need for windows for spectral analysis is eliminated, along with their distorting impact.

- The algorithm accepts non-evenly sampled time and frequency domain functions, for example the amplitude and phase samples at the logarithmic frequency scale

- The algorithm may be applied to signal segments of arbitrary length.

\section{REFERENCES}

[1] L. Ljung, System identification: Theory for the user. NJ: PrenticeHall, 1999.

[2] D. B. Percival and A. T. Walden. Spectral analysis for physical applications. Cambridge: Cambridge University Press, 1993.

[3] M. E. Cain, H. D. Ambos, F. X. Witkowski, and B. E. Sobel, "FastFourier transform analysis of signal-averaged electrocardiograms for identification of patients prone to sustained ventricular tachycardia”. Circulation, vol. 69, no.4, pp. 711-720, April 1984.

[4] R. Haberl, H. F. Schels, P. Steinbigler, G. Jilge, and G. Steinbeck, "Top-resolution frequency analysis of electrocardiogram with adaptive frequency determination". Circulation, vol. 82, no. 4, pp. 1183-1192, October 1990.

[5] D. Melkonian, Transients in Neuronal Systems (in Russian). Yerevan: Publishing House of Armenian Academy of Sciences, 1987.

[6] D. Melkonian, E. Gordon, C. Rennie, and H. Bahramali, "Dynamic spectral analysis of event-related potentials". Electroencephalogy clin. Neurophysiol., vol. 108, no.3, pp. 251-259, April 1998.

[7] D. Melkonian, T. Blumenthal, and E. Gordon, "Numerical Fourier transform spectroscopy of EMG half-waves: fragmentarydecomposition-based approach to nonstationary signal analysis". Biol. Cybern., vol. 81, no. 5-6, pp. 457-467, November 1999.

[8] D. Melkonian, E. Gordon, and H. Bahramali, "Single-event-related potential analysis by means of fragmentary decomposition". Biol. Cybern., vol. 85, no. 3, pp. 219-229, September 2001.

[9] T. D. Blumenthal, and D. Melkonian, "A model based approach to quantitative analysis of eyeblink EMG responses". J. Psychophysiol., vol. 17, no.1, pp. 1-11, 2003.

[10] D. Melkonian, T. D. Blumenthal, and R. Meares, "High resolution fragmentary decomposition - a model based method of nonstationary electrophysiological signal analysis". J. Neurosci. Methods, vol. 131, no. 1-2, pp. 149-159, December 2003.

[11] L. N. G. Filon, "On a quadrature formula for trigonometric integrals". Proc. R. Soc. Edinb., vol. 49, pp. 38-47, 1928.

[12] J. Schütte, "New fast Fourier transform algorithm for linear system analysis applied in molecular beam relaxation spectroscopy". Rev. Sci. Instrum., vol. 52, no.3, pp. 400-404, March 1981.

[13] S. Mäkinen, "New algorithm for the calculation of the Fourier transform of discrete signals". Rev. Sci. Instrum., vol. 53, no. 5, pp. 627-630, May 1982.

[14] R. Bousseljot, D. Kreiseler, and A. Schnabel, "Nutzung der EKGsignaldatenbank CARDIODAT der PTB über das Internet". Biomed. Tech., vol. 40, no. 1, p. 317, 1995.

[15] D. Kreiseler, and R. Bousseljot, "Automatisierte EKG-Auswertung mit Hilfe der EKG-Signaldatenbank CARDIODAT der PTB". Biomed Tech., vol. 40, no. 1, p.319, 1995.

[16] J. J. Rieta, F. Castells, C. Sanchez, V. Zarzoso, and J. Millet, "Atrial activity extraction for atrial fibrillation analysis using blind source separation". IEEE Trans. Biomed. Eng., vol. 51, no. 7, pp. 1176-1186, July 2004.

[17] S. Wu, Y. Qian, Z. Gao, and J. Lin, "A novel method for beat-tobeat detection of ventricular late potentials," IEEE. Trans. Biomed. Eng., vol. 48, no. 8, pp. 931-935, August 2001.

[18] J. C. Doyle, B. A. Francis, and A. R. Tannenbaum, Feedback control theory. New York: Macmillan, 1992.

[19] R. A. Serway, and J. W. Jewett, Physics for scientists and engineers. Brooks: Cole, 2003. 